\title{
The Mechanical Properties Analysis of Machine Tool Spindle After Remanufacturing Based on Uncertain Constraints
}

\author{
Ling Liu ${ }^{*}, 1,2$ \\ ${ }^{1}$ University of Xi'an in Shaan Xi, Xi'an 710065, China \\ ${ }^{2}$ School of Mechatronics, Northwestern Polytechnical University in Shanxi Xi'an 710072, China
}

\begin{abstract}
In this paper, the CNC machine spindle after remanufacturing is researched as an object on uncertain constraints. At first, the equations of the machine spindle motion based on beam theory are established. This article uses Finite Element Analysis (FEA) function to analyze the remanufacturing of machine spindle system in the free mode and while static and the actual working conditions of multi-modal analysis of the spindle's constraints state. By analysis it is known that the spindle vibrates and deforms at high speeds, and some assumptions are used to improve the unreasonable parameters, so that the spindle's dynamic performance is more stable and reliable in the conditions of the high speed and heavy load operation. In addition, simplifying the cost and shortening the design cycle are the part of the analysis. The results provides an optimized design and a basis for precision control for the heavy-duty mechanical spindle system or machine spindle system.
\end{abstract}

Keywords: Frequency domain analysis, parametric modeling, spindle modal, static characteristics..

\section{INTRODUCTION}

The electric spindle, magnetic suspension bearing, angular contact ball bearer, liquid hydrostatic bearing and air bearing represents the developmental trends of the spindle of the high-performance lathe in China [1, 2]. Features of the spindle are mainly studied by using the methods such as FEA method, centralized parameter method, transfer matrix method and boundary element method [3]. Bollinger processes the radial angular contact ball bearing as the radial spring and damper and establishes the beam model of the spindle of the lathe [4]. KOSMATKA establishes Timoshenko beam model based on the Hamilton principle and analyzes the influence of the axial force on stability of the beam [5]. Jorgensen and Shin establish the bearing loaddistortion model and studies influence of the thermal distortion on spindle rigidity and inherent frequency. CAO introduces centrifugal force, gyroscopic effect, pre-tightening force and rotor offset to Timoshenko beam model and lays foundation for further research on the spindle of the lathe [68]. In addition, experts and scholars have achieved some achievements in research on the spindle of the lathe in China. Guan Xiyou summarizes the dynamic research methods on the spindle of the lathe [9]. Xu Linfeng analyzes distortion of the spindle and guide of the lathe [10] and deduces force features of the spindle and guide. Zhang Yaomai simplifies the bearing support as spring dampness unit and establishes the dynamic finite element model of the spindle [11]. Yu Tianbiao analyzes high-speed spindle system of the grinding machine supported by the liquid hydrostatic bearing and studies the relation between the rotation rate of the spindle, system rigidity and inherence frequency [12].

Many unknown factors affect processing quality in actual processing, e.g. casting quality of semi-finished products and bearing life. Based on the actual production conditions, this paper mainly analyzes the influences of the bearing constraint position on the modes of the spindle. This paper first considers cutting influences, establishes the rigidity matrix of the spindle, and analyzes the vibration mode of the spindle by using the finite element analysis software. The analysis results are validated via the hammer trial to prove correctness of the analysis conclusions. The research conclusions can provide theoretical reference for the design of the spindle system of the lathe.

\section{ANALYSIS ON MILLING CUTTER FORCE}

The theoretical computing equation of the cutting force of the miller is described as:

$F_{t}=\cos (\beta-\gamma)=\tau_{s} \varepsilon^{n} a_{p} a_{w}[\operatorname{ctg} \varphi+\operatorname{tg}(\varphi+\beta-\gamma)]$

$\beta$-friction angle; $\gamma$-anterior angle; $\varphi$-cutting angle; $\tau_{s}$ —cutting strength; $\varepsilon$-distortion factor; $a_{p}$ —cutting depth; $a_{w}$-cutting width; From the cutting force computing model in the Fig. (1), the cutting force mainly includes circumferential cutting force $F_{t}$, radial cutting force $F_{r}$ and radial cutting force $F_{a}$. The circumferential cutting force and radial cutting force will make the spindle rotate. The axial cutting force will make the spindle generate the bending moment [13]. 


\section{ESTABLISH RIGIDITY MODEL OF SPINDLE}

\subsection{Model Simplification}

The spindle studied in this paper can be simplified as the structure in the Fig. (2). Based on the existing design parameters, it is known that $D_{1}=32 \mathrm{~mm}, D_{2}=132 \mathrm{~mm}$, $D_{3}=140 \mathrm{~mm}, D_{4}=65 \mathrm{~mm}, L_{I}=50 \mathrm{~mm}, L_{2}=100 \mathrm{~mm}, L_{3}=213$ $\mathrm{mm}$ and $L_{4}=453 \mathrm{~m}$. The spindle is made of 38CrMoAlA. The main parameters of this material are described as follows: modulus of elasticity is $2.1 \mathrm{e} 5 \mathrm{Mpa}$, yield strength is 835 $\mathrm{MPa}$, Poisson ratio is 0.3 and shear strength is $765 \mathrm{MPa}$. Generally the bearing is fully constrained in the traditional analysis on the spindle. This method ignores the influence of the elastic support on the inherent frequency of the spindle. It is difficult for the existing FEA method to combine the bearing force and spindle vibration. The bearing is simplified as the spring unit with the axial and radial rigidity in analysis. The spring rigidity can be selected by the type and size of the bearing, as shown in the Fig. (4). Based on the separate force analysis of the bearing, the forces on the spring unit are different in four directions during rotation of the spindle and the force reduces from down to up, as shown in the Fig. (3).

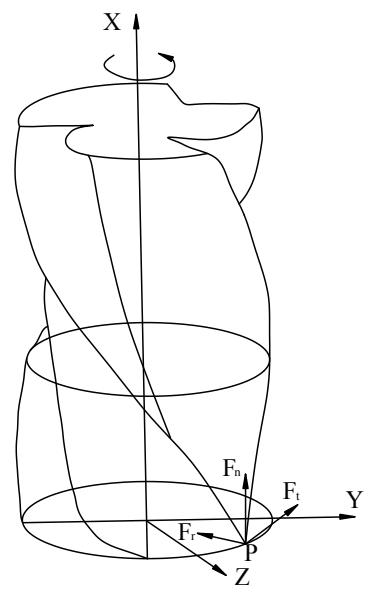

Fig. (1). Analysis and computing of milling force.

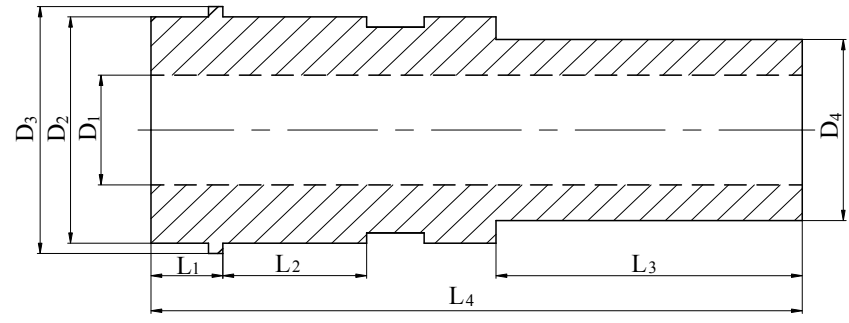

Fig. (2). Simplification model of spindle.

\subsection{Establish Basic Model}

For the shear axis, assuming the transverse fiber without extrusion, we can get

$\varepsilon_{y}=0, \quad \bar{u}=u=f(x)$

The radial shift at any point inside the axis is described as:

$\bar{u}=u-\theta y=f_{u}(x)-\theta$
The rotation angle $\theta$ is the derivative of the deflection in the equation (3). Based on the geometric equation, we can get:

$\gamma_{x y}=\frac{\partial \bar{u}}{\partial x}+\frac{\partial \bar{u}}{\partial y}=\frac{d u}{d x}-\theta$

Stress component $\varepsilon_{\mathrm{x}}$ is:

$\varepsilon_{x}=\frac{\partial \bar{u}}{\partial x}=\frac{d u}{d x}-y \frac{d \theta}{d x}$

The stress $\sigma_{\mathrm{x}}$ inside the axis is:

$\sigma_{x}=E \varepsilon_{x}$

The stress component is $\varepsilon_{x}=700 / 211 \approx 3.3$. In addition, the shear stress $\tau_{\mathrm{xy}}=\mathrm{G} \gamma_{\mathrm{xy}}$ should be considered. The factor $\gamma_{\mathrm{xy}}=185 / 828 \approx 0.22$.

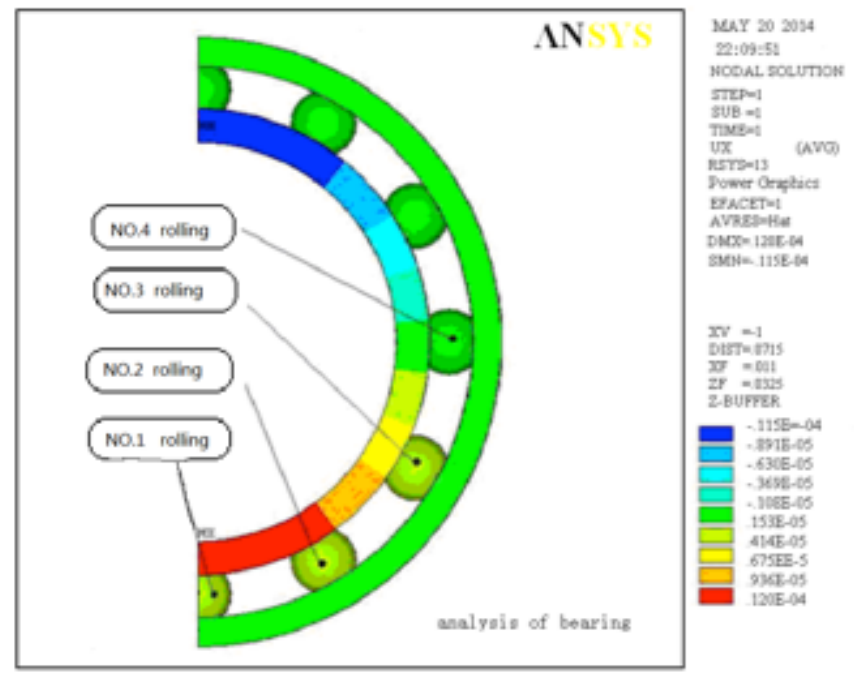

Fig. (3). Whole radial shift cloud diagram of bearing.

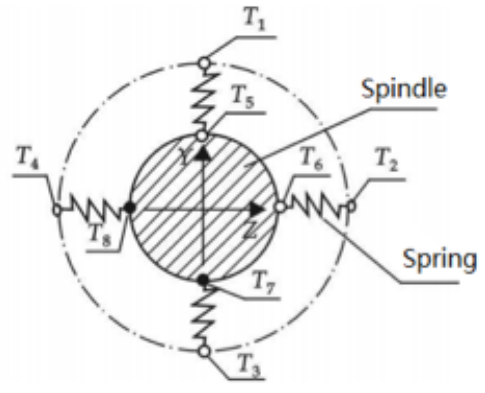

Fig. (4). Equivalent bearing distribution of spring.

\subsection{Influences of Shear}

Based on the above analysis, when the influences of the transverse shear distortion are not considered, equation (5) should be changed as:

$\varepsilon=\left\{\varepsilon_{x}\right\}=\left\{\frac{\partial \bar{u}}{\partial x}\right\}=\left\{\frac{d u}{d x}-y \frac{d^{2} u}{d x^{2}}\right\}=B_{b} a_{b}$

The stress matrix is:

$B_{b}=\left[N_{1}^{\prime}-y N_{3}^{\prime \prime}-y N_{4}^{\prime \prime} N_{2}^{\prime}-y N_{5}^{\prime \prime}-y N_{6}^{\prime \prime}\right]$ 
$\varepsilon_{x}=B_{b} a_{b}=\left[\begin{array}{llll}B_{1} & B_{2} & B_{3} & B_{4}\end{array}\right] a_{b}$, wherein $(\mathrm{i}=1,2,3, \ldots)$

$$
\left.B_{i}=\frac{1}{a b}\left[\begin{array}{cc}
b \frac{\partial N_{i}}{\partial \xi} & 0 \\
0 & a \frac{\partial N_{i}}{\partial \eta} \\
a \frac{\partial N_{i}}{\partial \eta} & b \frac{\partial N_{i}}{\partial \xi}
\end{array}\right]=\frac{1}{4 a b}\left[\begin{array}{lc}
b \xi_{i}\left(1+\eta_{o}\right) & 0 \\
0 & a \eta_{i}\left(1+\xi_{0}\right) \\
a \eta_{i}\left(1+\xi_{0}\right) & b \xi_{i}\left(1+\eta_{0}\right)
\end{array}\right]\right\}
$$

Assuming that the transverse shear distortion only affects the deflection, the additional shift field and additional axis deflection can be expressed as follows:

$u_{s}=\left\{\begin{array}{l}0 \\ v_{s}\end{array}\right\}, a_{s}=\left\{\begin{array}{c}v_{s 1} \\ v_{s 2}\end{array}\right\}$

$v_{\mathrm{s}}$ can be expressed as:

$\mathrm{v}_{s}=N_{1} \mathrm{v}_{s 1}+N_{2} \mathrm{v}_{s 2}$

To introduce the shift field into the plane geometric equation matrix, only the shear stress shown as the equation (13) is generated:

$\gamma_{x y}=\left[N_{1}^{\prime} N_{2}^{\prime}\right]\left\{\begin{array}{c}\mathrm{v}_{s 1} \\ \mathrm{v}_{s 2}\end{array}\right\}=B_{s} a_{s}$

Stress of axial unit is:

$\varepsilon=\left\{\begin{array}{l}\varepsilon_{x} \\ \gamma_{x y}\end{array}\right\}=\left\{\begin{array}{l}B_{b} a_{b} \\ B_{s} a_{s}\end{array}\right\}$

Unit stress is:

$\sigma=D \varepsilon=\left[\begin{array}{ll}E & 0 \\ 0 & G / k\end{array}\right]\left\{\begin{array}{l}\varepsilon_{x} \\ \gamma_{x y}\end{array}\right\}$

$G$ is the shear modulus in the equation (15). $k$ is the coefficient for non-uniform distribution of the shear stress. By referring to related information, $k=10 / 9$ for the round section.

\subsection{Unit Rigidity Matrix} get:

For the unit body, to use the virtual shift equation, we can

$\int \Omega^{\varepsilon} \delta \varepsilon^{T} \sigma d \Omega=\delta a^{e} F^{e}$

$\alpha^{\mathrm{e}}$ and $F^{\mathrm{e}}$ are the shift vector and force vector of the unit node in the equation (16) when bending and shearing are considered and $\alpha^{\mathrm{e}}$ can be expressed as the equation (17). $\mathrm{A}$ is the sparse matrix, shown as the equation (18):

$a^{e}=a_{b}+A a_{s}$

$A=\left[\begin{array}{llllll}0 & 1 & 0 & 0 & 0 & 0 \\ 0 & 0 & 0 & 0 & 1 & 0\end{array}\right]^{T}$

To substitute the equation (14), (15) and (17) into the equation (15), we can get: $\delta a^{e T}\left(K_{b} a^{e}-K_{b} A a_{s}\right)+$

$\delta a_{s}^{T}\left(K_{s} a_{s}+A^{T} K_{b} A a_{s}-A^{T} K_{b} a^{e}\right)=\delta a^{e T} F^{e}$

where is $K_{b}=\int \Omega^{e} E B_{b}{ }^{T} B_{b} d \Omega, \quad K_{s}=\int \Omega^{e}(G / k) B_{s}{ }^{T} B_{s} d \Omega$ 。

Based on the balance relation in the equation (20):

$Q=\frac{G A}{k} \gamma_{x y}=\frac{G A}{k} \frac{d u_{s}}{d x}=\frac{G A}{k}\left(u_{s 2}-u_{s 1}\right)$,

we can get:

$Q=\frac{d M}{d x}=\frac{d}{d x}\left(-E I \frac{d^{2} u}{d x^{2}}\right)=$

$-E I \frac{d^{3} u_{b}}{d x^{3}}=\frac{6 E I}{l^{3}}\left[2\left(u_{b 2}-u_{b 1}\right)-l\left(\theta_{1}+\theta_{2}\right)\right]$

$b=12 k^{*} E^{*} I /\left(\mathrm{G}^{*} \mathrm{~A} * 1^{2}\right), E$ is the elastic modulus and $E=2.11 \mathrm{e}$. $I$ is the sectional inertia moment, $G$ is the shearing moment and $G=8.28 \mathrm{e}$. $A$ is the section area.

$A=1 / 2 \pi r^{2}=0.5 \times 3.14 \times 64^{2}=6430 \mathrm{~mm}^{2}$.

To substitute values, we can get:

$b=\frac{12 \times 10 / 9 \times 211 \times 0.05 \times\left(65^{2}-32^{2}\right)}{828 \times 0.5 \times \pi \times 64^{2} \times 373^{2}}=0.03$

$u_{2}-u_{1}=u_{\mathrm{b} 2}-u_{\mathrm{b} 1}+u_{\mathrm{s} 2}-u_{\mathrm{s} 1}$, so we can get:

$u_{s 2}-u_{s 1}=\frac{b}{1+b}\left(u_{2}-u_{1}\right)-\frac{l b}{2(1+b)}\left(\theta_{1}+\theta_{2}\right)$

If the transverse shear distortion is considered, the unit rigidity matrix is:

$K^{e}=\left[\begin{array}{ccccrr}\frac{E A}{l} & 0 & 0 & -\frac{E A}{l} & 0 & 0 \\ 0 & \frac{12 E I}{(1+b) l^{3}} & \frac{6 E I}{(1+b) l^{2}} & 0 & -\frac{12 E I}{(1+b) l^{3}} & \frac{6 E I}{(1+b) l^{2}} \\ 0 & \frac{6 E I}{(1+b) l^{2}} & \frac{(4+b) E I}{(1+b) l} & 0 & -\frac{6 E I}{(1+b) l^{2}} & \frac{(2-b) E I}{(1+b) l} \\ -\frac{E A}{l} & 0 & 0 & \frac{E A}{l} & 0 & 0 \\ 0 & -\frac{12 E I}{(1+b) l^{3}} & -\frac{6 E I}{(1+b) l^{2}} & 0 & \frac{12 E I}{(1+b) l^{3}} & -\frac{6 E I}{(1+b) l^{2}} \\ 0 & \frac{6 E I}{(1+b) l^{2}} & \frac{(2-b) E I}{(1+b) l} & 0 & -\frac{6 E I}{(1+b) l^{2}} & \frac{(4+b) E I}{(1+b) l}\end{array}\right]$

After computing, we can get:

$$
K^{e}=\left[\begin{array}{lccccc}
0.4 & 0 & 0 & -0.4 & 0 & 0 \\
0 & 0.2 & 1 & 0 & -0.2 & 1 \\
0 & 1 & 0.2 & 0 & -1 & 0.6 \\
-0.4 & 0 & 0 & 0.4 & 0 & 0 \\
0 & -0.2 & 1 & 0 & 0.2 & -1 \\
0 & -0.2 & 0.3 & 0 & 1 & 0.6
\end{array}\right]
$$

The whole rigidity matrix of the axis unit is computed as follows: 


$$
\frac{E A}{1.92} \times\left[\begin{array}{lccc}
1.4 & 0 & -1 & -0.2 \\
0 & 1.4 & -0.4 & 0.4 \\
-1 & -0.4 & 1.8 & 0.6 \\
-0.2 & 0.4 & 0.62 & 0.4
\end{array}\right]\left\{\begin{array}{l}
u_{1} \\
v_{1} \\
u_{2} \\
v_{2}
\end{array}\right\}=\sigma l\left\{\begin{array}{l}
1 \\
0 \\
0 \\
0
\end{array}\right\}
$$

The whole node shift of the unknown variant is

$a=\left[\begin{array}{llll}u_{1} & v_{1} & u_{2} & v_{2}\end{array}\right]^{T}=\sigma\left[\begin{array}{llllll}0.000539 & 0.0005292 & 0 & 0\end{array}\right]^{T}$.

By comparing the results of the solution of the variants obtained by using this model with results of the FEA, the results are found to be similar, so the scale can be used as the basis for preliminary design.

\section{MODE ANALYSIS}

Based on the Timoshenko beam model $m \ddot{q}+k \dot{q}=0, m$ is the mass matrix and $k$ is the rigidity matrix. By substituting the easy-to-know mass matrix and rigidity matrix established in the above section into the Timoshenko beam model, the vibration frequency of the spindle of the lathe can be obtained [14].

The analysis of the vibration of the simplified spindle model under free state was performed by using an FEA software. The first-order and second-order vibration is bending and third-order and fourth-order vibration is torsion, as shown in Fig. (5). The so-called free state indicates to the constrain freedom at one end of the spindle in five directions. Another end is the open end. The analysis results indicate that the third-order and fourth-order torsion amplitude is larger and is about 15 times of first-order and second-order torsion amplitude. The third-order and fourthorder vibration frequency is about 4 times of the first-order and second-order vibration frequency, so the spindle of the lathe cannot meet the usage requirement under free state.

To constrain the bearing at another end, generally the angular contact ball bearing is used as the spindle of the lathe. Only rotation in $\mathrm{Y}$ and $\mathrm{Z}$ axis is restricted in the simulation analysis. The analysis results indicate that the vibrational amplitude of the spindle will reduce after the bearing is constrained, as shown in Fig. (6). The bearing constraint is located at $30 \mathrm{~mm}$ inward at the constraint section and $100 \mathrm{~mm}$ inward at the free end. Based on the analysis of the constrained spindle, first-order and secondorder vibration amplitude is similar to 3-order and fourthorder vibration amplitude and reduces little compared to without the constraint, but the third-order and fourth-order vibration frequency speeds up. The increased frequency is very obvious. It indicates that the old vibration frequency of the spindle is increased after the bearing constraint is imposed, so the probability of self-excitation and forced vibration will reduce considerably.

\section{EXPERIMENT}

The dynamic features of the spindle are mainly studied by the hammering experiment method. The factor speed is not considered in our research, so the spindle can be fixed at the bearing constraint and one part of the spindle is (a) first-order frequency

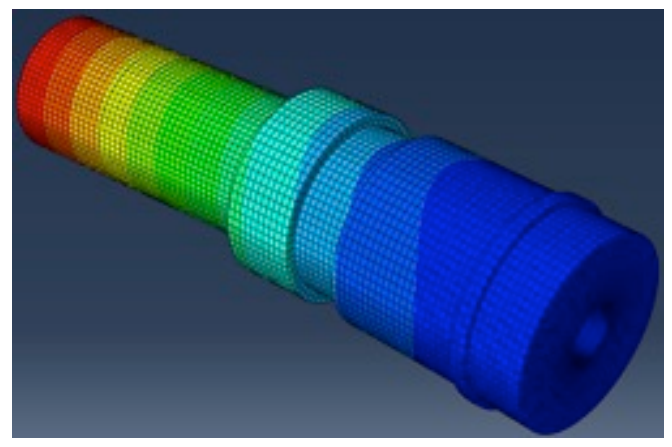

(b) second-order frequency

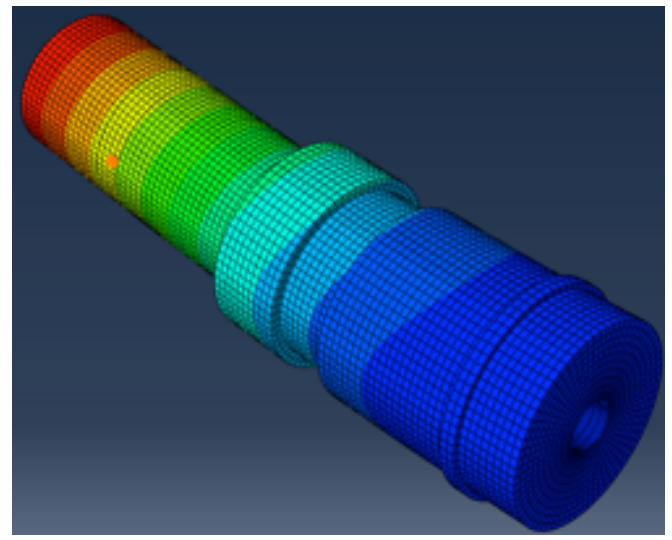

(c) Third-order frequency

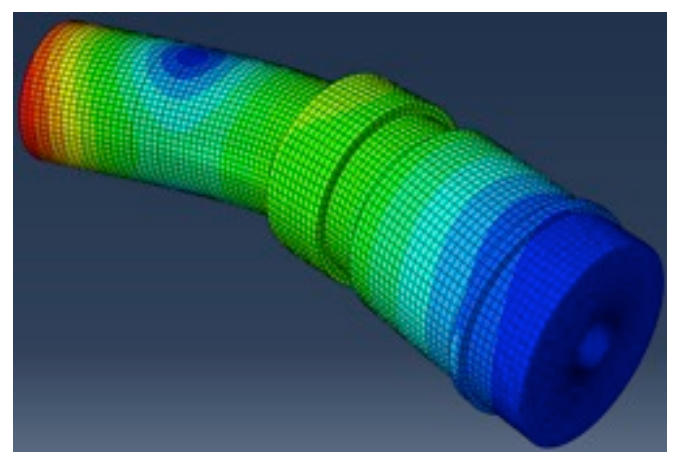

(d) Fourth-order frequency

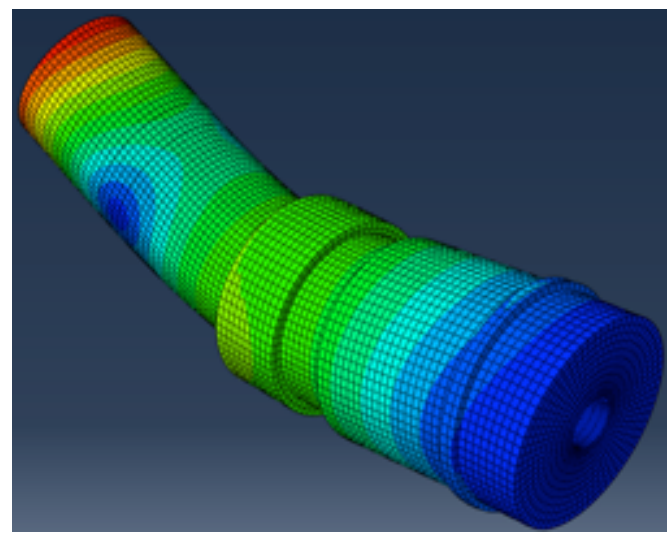

Fig. (5). Vibration frequency of spindle under free state.

hammered by using an iron hammer. The vibration frequency of the spindle is detected by using an advanced test instrument, as shown in Fig. (7). 
(a) First-order frequency

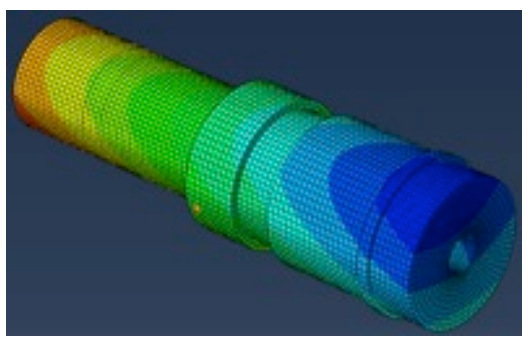

(b) Second-order frequency

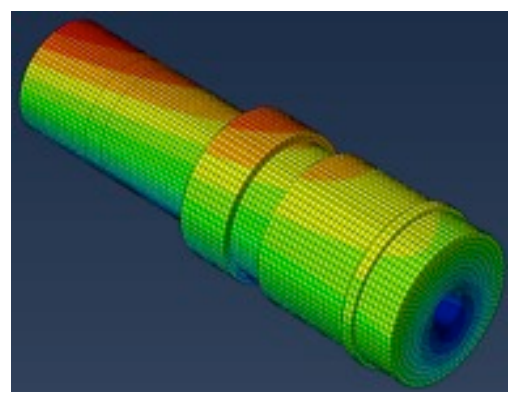

(c) Third-order frequency

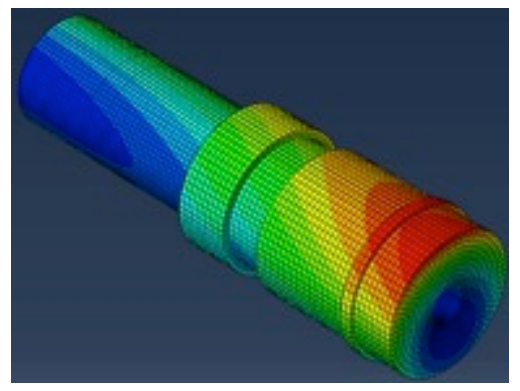

(d) Fourth-order frequency

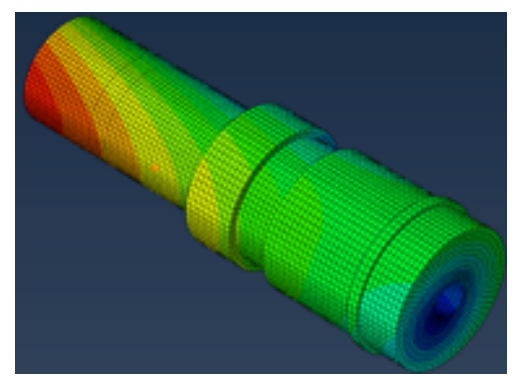

Fig. (6). Vibration frequency of spindle under bearing constraint state.
This experiment is mainly performed to detect the frequency of the spindle under fourth-order simulated vibration. The simulation data are similar to the experimental data, which indicates that the stablished model and modal analysis method are correct, but about $8 \%$ difference exist in the simulation data and experimental data due to the simplified model and simple test means. This error is within the acceptable range, as shown in Fig. (8).

\section{frequency}

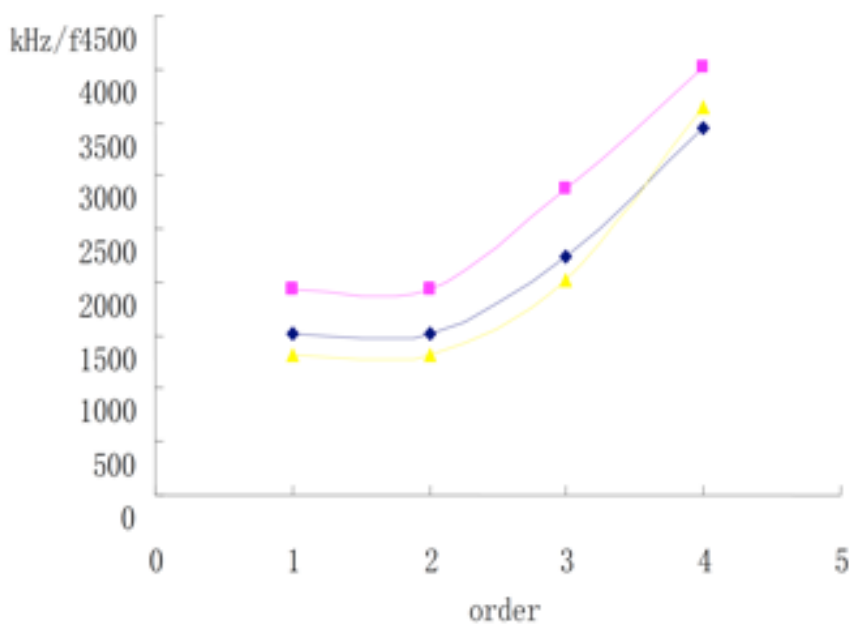

Fig. (8). Comparison of spindle frequency.

\section{CONCLUSION}

(1) This paper establishes the rigidity matrix equation of the spindle and provides the basis for manual computing of the spindle frequency;

(2) This paper analyzes the modulus of the spindle by using the FEA method, embodies the role of the bearing constraint intuitively, and further studies the influence factors of the spindle frequency.

(3) The simulation model is validated via the hammering experiment to prove validity of the model and provide references for design in future.

\section{CONFLICT OF INTEREST}

The author confirms that this article content has no conflict of interest.

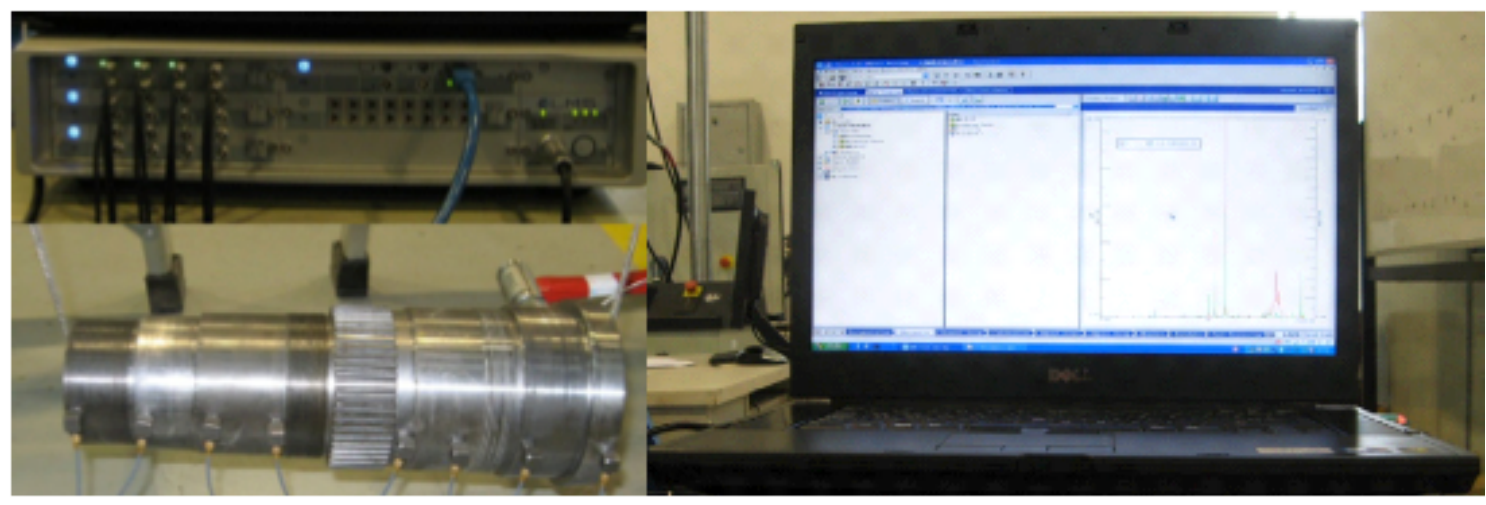

Fig. (7). LMS vibration test instrument. 


\section{ACKNOWLEDGEMENTS}

Thanks to the strong support of the Science and Technology Department of Shaanxi Province Project No. 2014K06-43! Thanks to the strong support of the Xi'an Science and Technology Project No. CXY1443WL24!

\section{REFERENCES}

[1] Q. He, "Research on cutting self-exited vibration control based on TK401 digital controlled lathe", Mechanical and Electric Engineering, vol. 24, no. 3, pp. 55-56, 2007.

[2] W. Xiong, "Analysis on current technical conditions of spindle of high-performance lathe in China", Metal Processing (Cold Processing), no. 18, pp. 6-11, 2011. Available from: www.metalworking 1950.com

[3] S. Gao, and G. Meng, "Research progress on dynamic features of lathe's spindle system", Vibration and Impact, vol. 26, no. 6, pp. 103-109, 2007.

[4] S.J. Zhang, and S. To, "The effects of spindle vibration on surface generation in ultra-precision raster milling international", Journal of Machine Tools \& Manufacture, no. 71, pp. 52-56, 2013.

[5] L. Yan, "Research on on-site measurement method and key technology of high-precision large bearing parts", Doctoral Dissertation of Shanghai University, April, 2008.

[6] U. V. Kumara, and T. L. Schmitzb, "Spindle dynamics identification for Receptance Coupling Substructure Analysis", Precision Engineering, no. 36, pp. 435-443, 2012.
[7] Z. Cui, "Optimization techniques and applications on the highspeed grinder design", Ph.d thesis, Hunan University, China, November 2010

[8] Y. Ma, "The energy characteristic state models for mechanical system conceptual design", Ph.d thesis, Dalian University of Technology, Septmber 2011.

[9] X. Guan, and W. Sun, "Research on dynamic feature analysis method of spindle system of numerical control machine", Combined Lathe and Automated Processing Technology, vol. 56, no. 4 , pp. 1-5, 2010.

[10] L. Wu, F. Guo, and L. Shang, "Analysis on distortion of lathe's spindle and guide", Journal of North China University of Water Resources and Electric Power, vol. 27, no. 4, pp. 52-54, 2006.

[11] Y. Zhang, C. Lui, and Z. Xie, "Einite element analysis of spindle component of high-speed lathe", Journal of Northeastern University (Natural Science Version), vol. 29, no. 10, pp. 14741477, 2008.

[12] T. Yu, X. Wang, and P. Guan, "Modulus analysis of spindle system of ultra-high-speed grinding and cutting lathe", Journal of Mechanical Engineering, vol. 48, no. 17, pp. 183-188, 2012.

[13] Y. Wang, "Research on Optimal Design of Lathe's Spindle", Master Dissertation of University of Electronic Science and Technology of China, 2009.

[14] L. Can, S. Ma, and Y. Zhao, "Finite element modeling and modulus analysis of spindle of heavy machine under multi-constraint state", Journal of Mechanical Engineering, vol. 48, no. 3, pp. 165-172, 2012.

(C) Ling Liu; Licensee Bentham Open.

This is an open access article licensed under the terms of the Creative Commons Attribution Non-Commercial License (http://creativecommons.org/licenses/by-nc/3.0/) which permits unrestricted, non-commercial use, distribution and reproduction in any medium, provided the work is properly cited. 